\title{
Disturbance Observer-based Predictive Repetitive Control with Constraints
}

\author{
Liuping Wang ${ }^{\mathrm{a}}$, Chris Freeman ${ }^{\mathrm{b}}$, Eric Rogers ${ }^{\mathrm{b}}$ \\ ${ }^{a}$ School of Engineering, RMIT University, Victoria 3000 Australia, (e-mail: liuping.wang@ rmit.edu.au) \\ ${ }^{b}$ Department of Electronics and Computer Science, University of Southampton, UK, (e-mail: $\{c f$, etar $\left.\} @ e c s . s o t o n . a c . u k\right)$
}

\begin{abstract}
This paper develops an observer-based predictive repetitive control system to track periodic reference signals or reject disturbances with band limited frequency content. The new design complements exist-ing approaches to predictive control, where a model of the periodic disturbance is embedded in the controller. In particular, the new design, based on a novel combination of repetitive control and a disturbance observer, results in a significant improvement in design transparency and implementation simplicity. Although the design is undertaken using a state-space-model, frequency response analysis based on the sensitivity and complementary functions is used to demonstrate the characteristics of the repetitive control system for disturbance rejection, reference following and measurement noise attenuation. Moreover, operational constraints can be included in the design for applications where this feature is required. Simulation studies are given to highlight the closed-loop performance achievable in the presence of constraints.Experimental validation results from application to a two-joint robotic arm are also given and discussed.
\end{abstract}

Keywords: predictive repetitive control, disturbance estimation, observer, robot arm

\section{INTRODUCTION}

Repetitive control (RC) is a well-established design method for tracking periodic signals and/or rejecting periodic disturbances, see, e.g. Hara et al. (1988) and exploits the internal model principle, see, e.g. (Francis and Wonham, 1976). In RC design, the plant input signal, considering the single-input single-output case for ease of presentation, is commonly generated by a controller described in transfer-function form, see, e.g. (Hara et al., 1988). If the reference signal has multiple-frequency content then by the internal model principle, these must be included as modes in the controller. The number of frequencies is proportional to the period and inversely proportional to the sampling interval. The result can be a very high order control system, especially under fast sampling, which, in turn, could lead to numerical sensitivity, noise amplification, sensitivity to modelling errors and other undesirable phenomena commonly encountered in practical applications.

A transparent way of limiting the controller order is based on selecting the dominant frequencies in the reference and/or disturbance signal and including only these in the controller. One method that can be used is frequency sampling filter models, see, e.g. Bitmead and Anderson (1981). Given the dominant frequencies, a repetitive control design has been developed where, as in model predictive control, operational constraints can be imposed if required. This approach to RC design has been termed repetitive predictive design. Preliminary application results on this approach were reported in, e.g. (Wang et. el, 2012) and followed through to comprehensive experimental validation on a 2-joint robot (Wang et al., 2013, 2016), industrial electrical drives and a power converter (Wang et al., 2015). (A tutorial overview of this approach to control design can be found in (Wang, 2016)).

This paper develops an alternative repetitive predictive design which is based on estimating the periodic disturbance signal using a suitably structured observer, and then subtracting it from an optimized control signal. This novel approach provides significant advantages in terms of implementational efficiency, design transparency and ease of analysis. This approach is related to control system design using an extended disturbance observer, and constitutes its first application to the field of RC. Such an approach has been developed for engine-induced vibrations in the 
context of estimation of the sinusoidal disturbances and their cancelation (Bohn et al., 2004). In (Bodson., 2005; Jia., 2009), an adaptive frequency estimation technique is incorporated into traditional disturbance observer methods to estimate the disturbance frequency online. A transfer-function based approach using a disturbance observer for controlling magnetic disk drives was developed in (White et al., 2000). A similar approach was used together with online estimation of disturbance frequency in (Zhen and Tomizuka., 2008). The methods for analysis and design of extended disturbance observers were surveyed in a recent paper (Madońsk and Herman., 2015).

The next section of this paper develops the new disturbance observer-based control system design using as a setting for analysis state-space and disturbance models obtained from previous work, e.g., (Wang et al., 2010, 2012, 2013, 2016). The control system is then developed in the following section using a model predictive control setting that enables operational constraints to be included. Sections describing experimental evaluation of the design on a two-input two-output robotic system executing a pick and place task and conclusions and further work, respectively, complete the paper.

\section{DISTURBANCE OBSERVER based PREDICTIVE-REPETITIVE CONTROL SYSTEM DESIGN}

Let $q^{-1}$ denote the backward shift operator. Then a standing assumption is that a disturbance model, denoted by $D\left(q^{-1}\right)$, is available that represents the frequency characteristics of the signal considered. In some cases, this model is immediate from the signal description, e.g., for a known piece-wise constant reference signal $D\left(q^{-1}\right)=1-q^{-1}$. If $D\left(q^{-1}\right)$ cannot be constructed from knowledge of the signal then methods have been developed including the frequency sampling filters (Bitmead and Anderson, 1981) discussed in the previous section. Recent experimental validation of this approach can be found in (Wang et al., 2016).

The plant to be controlled is assumed to have the same number, $m$, of inputs and outputs, and state-space model

$$
\begin{aligned}
x_{m}(k+1) & =A_{m} x_{m}(k)+B_{m} u(k), \\
y(k) & =C_{m} x_{m}(k),
\end{aligned}
$$

where $x_{m}(k)$ is the $n_{1} \times 1$ state vector, $u(k)$ is the $m \times 1$ input vector, $y(k)$ is $m \times 1$ output vector. For design, the state-space model matrices $\left(A_{m}, B_{m}, C_{m}\right)$ together with $D\left(q^{-1}\right)$ are assumed to be available. A number of ways exist of satisfying the internal model principle for this case and the one used here is to add a vector to the state dynamics and then write a state-space model for the resulting dynamics and use this resulting model for analysis.

Let $\mu(k)$ is a vector that has the same dimension as the control signal and in the case of a single disturbance or reference signal chose $i$ th entry as

$$
\mu_{i}(k)=\frac{\varepsilon_{i}(k)}{D\left(q^{-1}\right)}
$$

where $\varepsilon_{i}(k)$ is a white noise sequence with zero mean and variance $\sigma_{i}, 1 \leq i \leq m$. For more than one disturbance and/or reference signal, the frequency sampling filter analysis is applied to each signal, the least common denominator of the resulting polynomials is computed and forms $D(z)$ in this last equation. The term $B_{m} \mu(k)$ is then added to the state equation in (1).

The polynomial $D\left(q^{-1}\right)$ is assumed to contain the dominant modes in the corresponding signal(s). Assuming that it is of degree $\gamma$, this polynomial can be written as

$$
D\left(q^{-1}\right)=1+d_{1} q^{-1}+d_{2} q^{-2}+d_{3} q^{-3}+\ldots+d_{\gamma} q^{-\gamma}
$$

Moreover, write $\mu(k+1)$ as

$$
\begin{aligned}
\mu(k+1)= & -d_{1} \mu(k)-d_{2} \mu(k-1)-\ldots- \\
& -d_{\gamma-1} \mu(k-\gamma+2)-d_{\gamma} \mu(k-\gamma+1)+\varepsilon(k)
\end{aligned}
$$


where the compatibly dimensioned vector $\varepsilon(k)$ has as each entry zero mean white noise. Also introduce

$$
z(k)=\left[\begin{array}{llll}
x_{m}^{T}(k) & \mu^{T}(k) & \ldots & \mu^{T}(k-\gamma+1)
\end{array}\right]^{T} .
$$

Then observer design will be based on the following state-space model

$$
\begin{aligned}
z(k+1) & =A_{o} z(k)+B_{o} u(k)+\bar{B}_{o} \varepsilon(k) \\
y(k) & =C_{o} z(k)
\end{aligned}
$$

where

$$
\begin{gathered}
A_{o}=\left[\begin{array}{cc}
A_{m} & \bar{B}_{m} \\
0 & A_{d}
\end{array}\right], \\
A_{d}=\left[\begin{array}{ccccc}
-d_{1} I & -d_{2} I & \ldots & -d_{\gamma-1} I & -d_{\gamma} I \\
I & 0 & 0 & \ldots & 0 \\
0 & I & 0 & \ldots & 0 \\
\vdots & \ddots & \ddots & \ddots & \vdots \\
0 & 0 & \ldots & I & 0
\end{array}\right] . \\
\bar{B}_{m}=\left[\begin{array}{cc}
B_{m} & 0
\end{array}\right]
\end{gathered}
$$

where for the remainder of this paper 0 and $I$ denote the compatibley dimensioned null and identity matrices, respectively, and

$$
\begin{gathered}
B_{o}=\left[\begin{array}{c}
B_{m} \\
0
\end{array}\right], \bar{B}_{o}=\left[\begin{array}{l}
0 \\
I
\end{array}\right] \\
C_{o}=\left[\begin{array}{ll}
C_{m} & O
\end{array}\right]
\end{gathered}
$$

Observability of the pair $\left(A_{o}, C_{o}\right)$ follows if this property holds for $\left(A_{m}, C_{m}\right)$. To estimate the augmented state vector $z(k)$, suppose that an observer gain matrix $K_{o b}$ is chosen such that the closed-loop observer error system state matrix $\left(A_{o}-K_{o b} C_{o}\right)$ is stable with a desired response speed. Then the augmented state vector $z(k)$ is estimated using

$$
\hat{z}(k+1)=A_{o} \hat{z}(k)+B_{o} u(k)+K_{o b}\left(y(k)-C_{o} \hat{z}(k)\right),
$$

and the estimated disturbance vector $\hat{\mu}(k)$ is obtained. In many applications, the state vector entries can be measured, e.g. in electrical drives and power converters, the current and voltage variables are measured by the respective sensors. In such cases, only the disturbance vector $\mu(k)$ requires estimation and reduces the complexity of the design (arguably More relevant) implementation. This case is considered in the remainder of this paper, starting with the observer design.

Using (1) and (2) results in

$$
\begin{aligned}
y(k+1) & =C_{m} x_{m}(k+1) \\
& =C_{m} A_{m} x_{m}(k)+C_{m} B_{m} u(k)+C_{m} B_{m} \mu(k)
\end{aligned}
$$

and hence

$$
C_{m} B_{m} \mu(k)=y(k+1)-C_{m} A_{m} x_{m}(k)-C_{m} B_{m} u(k)=\zeta(k)
$$

and the estimation of $\mu(k)$ without estimating the state vector $x_{m}(k)$ is through the system

$$
\begin{aligned}
\mu(k+1) & =A_{d} \mu(k)+\varepsilon(k) \\
\zeta(k) & =C_{m} B_{m} \mu(k)
\end{aligned}
$$


Assume that (9) is observable and that an observer gain matric $K_{o b}^{r}$ has been designed such that the resulting observer error dynamics are stable. Then the $\mu(k)$ is estimated using the following observer structure :

$$
\begin{aligned}
\hat{\mu}(k+1) & =A_{d} \hat{\mu}(k)+K_{o b}^{r}\left(\zeta(k)-C_{m} B_{m} \hat{\mu}(k)\right) \\
& =A_{d} \hat{\mu}(k)+K_{o b}^{r}(y(k+1) \\
& \left.-C_{m} A_{m} x_{m}(k)-C_{m} B_{m} u(k)-C_{m} B_{m} \hat{\mu}(k)\right) .
\end{aligned}
$$

This observer requires $y(k+1)$, which is one step ahead of the measurement at the sampling instant $k$, which complicates its implementation. Alternatively, introduce

$$
\hat{\beta}(k)=\hat{\mu}(k)-K_{o b}^{r} y(k)
$$

and add and subtract the term $\left(A_{d}-K_{o b}^{r} C_{m} B_{m}\right) K_{o b}^{r} y(k)$ from the left-hand side of (11) followed by routine manipulations enable (11) to be written as

$$
\begin{aligned}
\hat{\beta}(k+1) & =\left(A_{d}-K_{o b}^{r} C_{m} B_{m}\right) \hat{\beta}(k)+\left(A_{d}-K_{o b}^{r} C_{m} B_{m}\right) K_{o b}^{r} y(k), \\
& -K_{o b}^{r} C_{m} A_{m} x_{m}(k)-K_{o b}^{r} C_{m} B_{m} u(k) .
\end{aligned}
$$

This last observer equation can be implemented where at $k=0$, an initial condition for $\hat{\beta}(0)$ is selected and the disturbance $\hat{\mu}(0)=\hat{\beta}(0)+K_{o b}^{r} y(0)$. Together with the measurements of states, outputs, and control signals at sampling instant $k$, the estimation of $\hat{\beta}(k+1)$ is performed. Additionally, $\hat{\mu}(k)=\hat{\beta}(k)+K_{o b}^{r} y(k)$ is calculated for repetitive predictive control system.

\section{Control System Design}

Introduce

$$
\tilde{u}(k)=u(k)+\mu(k)
$$

and then (1) can be written as

$$
x_{m}(k+1)=A_{m} x_{m}(k)+B_{m} \tilde{u}(k) .
$$

Suppose also the pair $\left(A_{m}, B_{m}\right)$ is controllable, then there exists a control law $\tilde{u}(k)=-B_{m} K x_{m}(k)$ such that

$$
x_{m}(k+1)=\left(A_{m}-B_{m} K\right) x_{m}(k)
$$

is stable. Hence the future trajectory of the control vector $\tilde{u}(k)$ can be modelled using a sequence of the pulse functions or a set of Laguerre functions (Wang, 2009) since, for the stable system described by (15) with bounded initial conditions, $\lim _{k \rightarrow \infty} \tilde{u}(k) \rightarrow 0$. This step is essential if a limited number of parameters are to be used to parameterize the future control trajectories in model predictive control design.

The current plant information at any sample instant is given in the vector $k x_{m}(k)$ whose construction was detailed in the previous section. Also define the future optimal control vector $\tilde{U}$ as

$$
\tilde{U}=\left[\begin{array}{llll}
\tilde{u}(k)^{T} & \tilde{u}(k+1)^{T} & \ldots & \tilde{u}\left(k+N_{c}-1\right)^{T}
\end{array}\right]^{T}
$$

where $N_{c}$ is the control horizon, i.e., the number of parameters used to describe the future control trajectory. Given this information, the future state vectors are predicted for $N_{p}$ samples, where $N_{p}$ is the prediction horizon and $N_{c} \leq N_{p}$. Suppose also that after $N_{c}$ samples, the control vector $\tilde{u}(k)=0$ for $k \geq N_{c}$ and write the resulting state vectors as

$$
X=\left[\begin{array}{lll}
x_{m}(k+1 \mid k)^{T} & \ldots & x_{m}\left(k+N_{p} \mid k\right)^{T}
\end{array}\right]^{T} .
$$


Using (14), the future state vectors are, given $\mathrm{s} U_{s}$, computed as

$$
X=F_{x} x_{m}(k)+\Phi_{s} \tilde{U}
$$

where

$$
F_{x}=\left[\begin{array}{c}
A_{m} \\
A_{m}^{2} \\
\vdots \\
\vdots \\
A_{m}^{N_{p}}
\end{array}\right] \Phi_{s}=\left[\begin{array}{cccc}
B_{m} & 0 & . . & 0 \\
A_{m} B_{m} & B_{m} & . . & 0 \\
A_{m}^{2} B_{m} & A_{m} B_{m} & . . & 0 \\
\vdots & \vdots & . . & \vdots \\
A_{m}^{N_{p}-1} B_{m} & A_{m}^{N_{p}-2} B_{m} & . . & A_{m}^{N_{p}-N_{c}} B_{m}
\end{array}\right]
$$

The design criterion for the predictive repetitive controller is to find $\tilde{U}$ to minimize the cost function

$$
J=X^{T} \bar{Q} X+\tilde{U}^{T} \bar{R} \tilde{U}
$$

where $\bar{Q}$ is a block diagonal matrix with identical block diagonal matrix entries $Q$, where $Q$ is a symmetric positive semi-definite matrix and $\bar{R}$ is block diagonal matrix with identical block diagonal matrix entries $R$, where $R$ is a positive definite matrix.

Combining (16) and the cost function gives

$$
\begin{aligned}
J= & \tilde{U}^{T}\left(\Phi_{s}^{T} \bar{Q} \Phi_{s}+\bar{R}\right) \tilde{U}+2 \tilde{U}^{T} \Phi_{s}^{T} \bar{Q} F_{x} x_{m}\left(k_{i}\right) \\
& +x_{m}(k)^{T} F_{x}^{T} Q F_{x} x_{m}(k) .
\end{aligned}
$$

and the solution of this optimal control problem is

$$
\tilde{U}=-\left(\Phi_{s}^{T} \bar{Q} \Phi_{s}+\bar{R}\right)^{-1} \Phi_{s}^{T} \bar{Q} F_{x} x_{m}(k) .
$$

If the state vector $x_{m}(k)$ is not measurable, the observer specified by (6) is used to estimate the augmented state vector $\hat{z}\left(k\right.$, from which $\hat{x}_{m}(k)$ is obtained and used instead of $x_{m}\left(k_{i}\right)$. The actual control vector at sampling instant $k$ is given by

$$
u(k)=\tilde{u}(k)-\hat{\mu}(k)
$$

where $\hat{\mu}(k)$ is calculated using the observer of (6) or (13).

In this design the disturbance model is embedded through the observer and therefore the reference vector should enter the system as an output disturbance with a negative sign. Otherwise, the control system will have steady-state errors when used for reference tracking. Given the reference vector $r(k)$, the observer $(6)$ can be rewritten as

$$
\hat{z}(k+1)=A_{o} \hat{z}(k)+B_{o} u(k)+K_{o b}\left(y(k)-r(k)-C_{o} \hat{z}(k)\right)
$$

if the state vector $x_{m}(k)$ can be measured, the reference vector enters the system through the reduced order observer as

$$
\begin{aligned}
\hat{\mu}(k) & =\hat{\beta}(k)+K_{o b}^{r}(y(k)-r(k)) \\
\hat{\beta}(k+1) & =\left(A_{d}-K_{o b}^{r} C_{m} B_{m}\right) \hat{\beta}(k) \\
& +\left(A_{d}-K_{o b}^{r} C_{m} B_{m}\right) K_{o b}^{r}(y(k)-r(k)) \\
& -K_{o b}^{r} C_{m} A_{m} x_{m}(k)-K_{o b}^{r} C_{m} B_{m} u(k) .
\end{aligned}
$$

\subsection{Constrained Design}

This section extends the design of the previous section to include input and output operational constraints in the design. The method used leads to a real-time optimization problem. The central idea is to minimize the objective 
function $J$ as in (17) subject to linear inequality constraints.

If an integrator is to be included in the observer, the input constraints are somewhat more complicated because they will involve the estimated disturbance $\hat{\mu}(k)$. For example, a control amplitude constraint can be written as

$$
u^{\min } \leq u(k) \leq u^{\max }
$$

or, in terms of $\tilde{u}(k)$,

$$
u^{\min }+\hat{\mu}(k) \leq \tilde{u}(k) \leq u^{\max }+\hat{\mu}(k)
$$

and the rate of change of the input as

$$
\Delta u^{\min }+u(k-1)+\hat{\mu}(k) \leq \tilde{u}(k) \leq \Delta u^{\max }+u(k-1)+\hat{\mu}(k .
$$

Also $\tilde{u}(k)$ is the first entry in $\tilde{U}$, these input constraints are converted into inequality constraints in terms of $\tilde{U}$.

The constraints for the output or states are formulated using (16). Once the constraints are formulated, a quadratic programming algorithm can be used to solve the constrained predictive control problem.

\section{Experimental evaluation}

The preceeding control design has been implemented on an anthropomorphic robot arm performing a 'pick and place' task in a horizontal plane using two joints, as shown in Fig. 1. Its end-effector travels between the pick and place locations along a straight line using joint reference trajectories which minimize the acceleration of the endeffector. After reaching the 'place' location, the robot then returns back to the starting 'pick' location. The overall two-input, two-output system model has been identified from frequency domain test data, and is described by the transfer-function matrix given in (23).

$$
\left[\begin{array}{l}
y_{1} \\
y_{2}
\end{array}\right]=\left[\begin{array}{l}
G_{11} G_{12} \\
G_{21} G_{22}
\end{array}\right]\left[\begin{array}{l}
u_{1} \\
u_{2}
\end{array}\right]
$$

$$
\begin{gathered}
G_{11}(s)=\frac{0.16 s^{9}+14.51 s^{8}+578.2 s^{7}+1.392 e 4 s^{6}+2.26 e 5 s^{5}+2.58 e 6 s^{4}+2.09 e 7 s^{3}+1.17 e 8 s^{2}+4.21 e 8 s+7.6 e 8}{5.25 e-5 s^{12}+0.01463 s^{11}+0.91 s^{10}+31.2 s^{9}+714.1 s^{8}+1.19 e 4 s^{7}+1.45 e 5 s^{6}+1.4 e 6 s^{5}+1.01 e 7 s^{4}+5.7 e 7 s^{3}+2.3 e 8 s^{2}+5.9 e 8 s+7.6 e 8}, \\
G_{12}(s)=\frac{-0.022 s^{7}-3.24 s^{6}-88.3 s^{5}-1347 s^{4}-1.06 e 4 s^{3}-4.52 e 4 s^{2}}{5.25 e-5 s^{1} 0+0.014 s^{9}+0.72 s^{8}+20 s^{7}+363 s^{6}+4645 s^{5}+4.3 e 4 s^{4}+2.9 e 005 s^{3}+1.4 e 6 s^{2}+4.18 e 6 s+6.323 e 6}, \\
G_{21}(s)=\frac{-0.16 s^{7}-8.7 s^{6}-194 s^{5}-2498 s^{4}-1.78 e 4 s^{3}-6.64 e 4 s^{2}}{5.25 e-5 s^{10}+0.014 s^{9}+0.67 s^{8}+17.9 s^{7}+316 s^{6}+3963 s^{5}+3.6 e 4 s^{4}+2.42 e 5 s^{3}+1.1 e 6 s^{2}+3.5 e 6 s+5.3 e 6}
\end{gathered}
$$

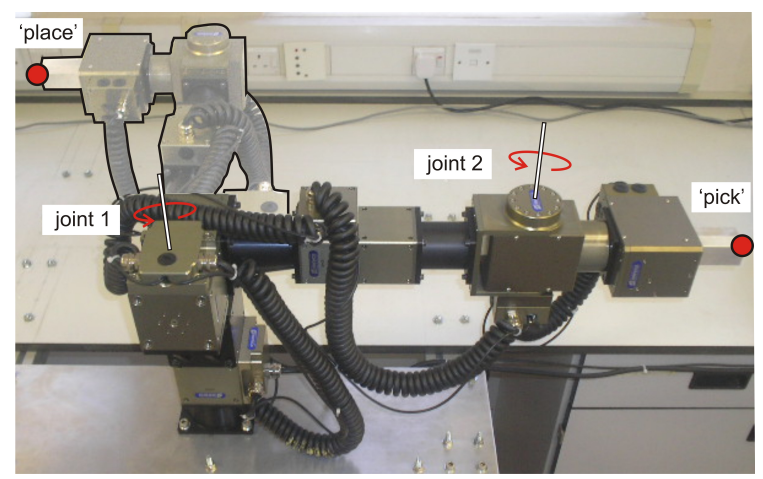

Figure 1: Anthropomorphic robot arm showing pick and place locations. 


\begin{tabular}{|c||r|}
\hline$N_{p}$ & 20 \\
\hline$N_{c}$ & 6 \\
\hline$r_{c}$ & 0.1 \\
\hline$r_{o b}$ & 10000 \\
\hline
\end{tabular}

Table 1: Choice of performance parameters

The continuous-time system is sampled using the sampling interval $\Delta t=0.05(\mathrm{sec})$. The reference trajectories for output $y_{1}$ and $y_{2}$ are, respectively, shown in the upper plots of Figures $2 \mathrm{a}$ ) and $\mathrm{b}$ ), and the control objective is for each output to follow the corresponding reference signal as closely as possible in presence of measurement noise and model uncertainty. The reference signals for the outputs $y_{1}$ and $y_{2}$ each have a period of 20 seconds. With the sampling interval chosen to be 0.05 (sec), the number of samples for each period is $\frac{20}{0.05}=400$. From previous analysis (see (Wang et al., 2013)), the polynomial $D\left(z^{-1}\right)$ is selected as

$$
\begin{aligned}
D(z)= & \left(1-z^{-1}\right)\left(1-2 \cos \left(\frac{2 \pi}{400}\right) z^{-1}+z^{-2}\right) \times \\
& \left(1-2 \cos \left(\frac{4 \pi}{400}\right) z^{-1}+z^{-2}\right)
\end{aligned}
$$

In this case, when the experimentally determined transfer-function matrix entries are used to construct a statespace model, the resulting state variables have no physical meaning. Thus, a full order observer is required to estimate both the state vector $x_{m}(k)$ and the disturbance $\mu(k)$. In the design of the observer, we $Q_{o b}=I$ and $R_{o b}=r_{o b} I$ with $r_{o b}$ being adjustable has been used. For the predictive repetitive controller design, $Q=C_{m}^{T} C_{m}$ and $R=r_{c} I$ with $r_{c}$ being adjustable was selected.. Table 1 shows the performance parameters used in the predictive repetitive control system design.

The performance parameters for the observer must be chosen carefully to ensure satisfactory performance. For instance, increasing the parameter $r_{o b}$ produces improved tracking accuracy, as confirmed by the experimental results shown in Figure 3. However, when the weighting coefficient $r_{o b}$ is reduced to 1000, the closed-loop predictive repetitive control system becomes unstable as shown in Figure 4. When the parameter $r_{o b}$ is reduced, the dynamic response speed of the observer error system is increased, which consequently reduces the robustness of the observer error system because there are inevitable modelling errors in the robotic system. However, when the weighting coefficient $r_{o b}$ is reduced to 1000, the closed-loop predictive repetitive control system becomes unstable as shown in Figure 4. When the parameter $r_{o b}$ is reduced, the dynamic response speed of the observer error system is increased, which consequently reduces the robustness of the observer error system because there are inevitable modelling errors in the robotic system.

\section{CONCLUSIONS}

This paper has developed a novel observer based predictive repetitive control system design which establishes the use of disturbance observers within RC. This design is based on the assumption of the existence of a input periodic disturbance and an observer is designed to estimate such a periodic disturbance. The structure embeds both design transparency and implementational efficiency compared with exisiting alternative approaches. Together with the model predictive controller, the resultant control system is shown experimentally to have the capability to track complex reference signals.

Goodwin, G. C., Graebe, S., Salgado, M., 2000. Control System Design. Prentice-Hall, Englewood Cliffs, NJ.

Francis, B., Wonham, W., 1976. The internal model principle of control theory. Automatica 12, 457-465. 

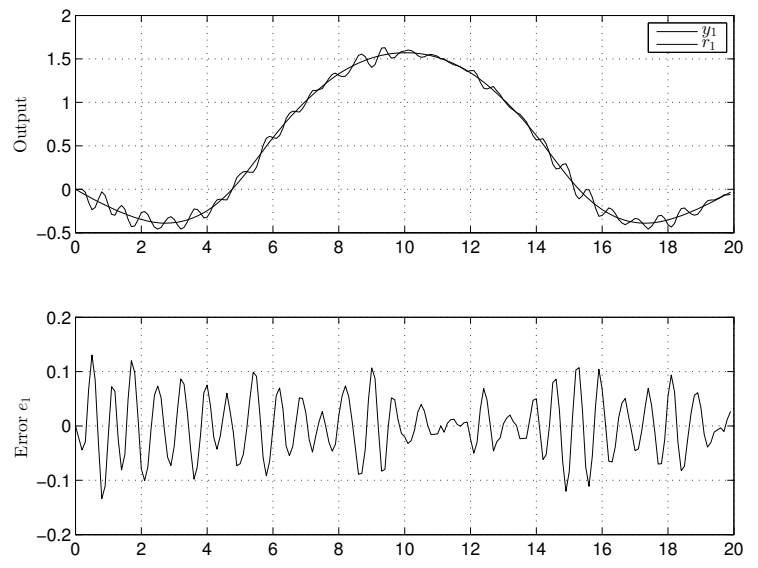

(a) $y_{1}$ and error
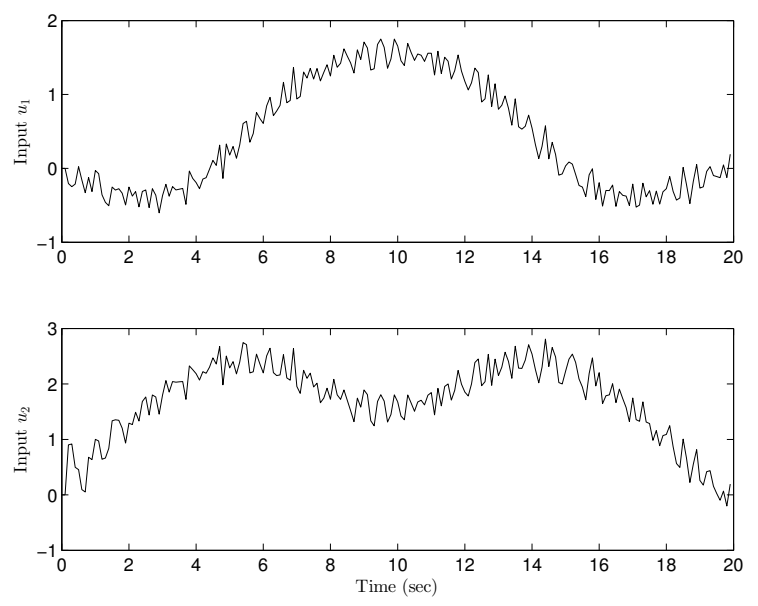

(c) Control signals
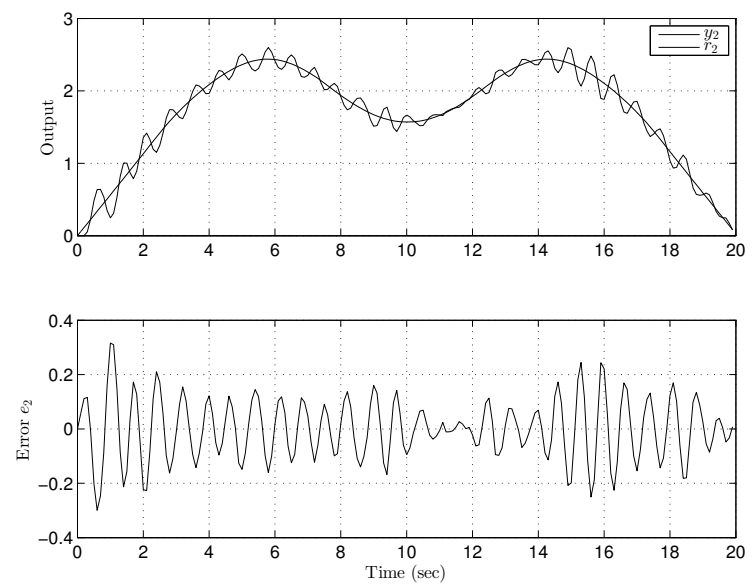

(b) $y_{2}$ and error

Figure 2: Experimental results using the performance parameters in Table 1. 

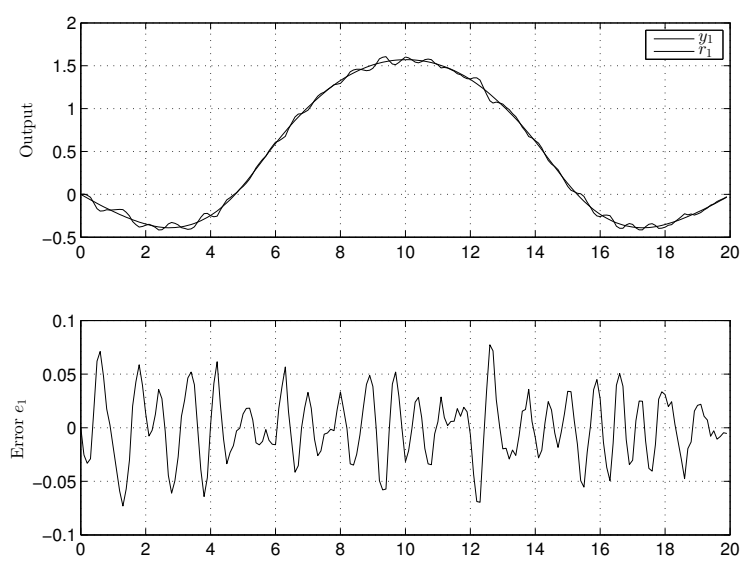

(a) $y_{1}$ and error
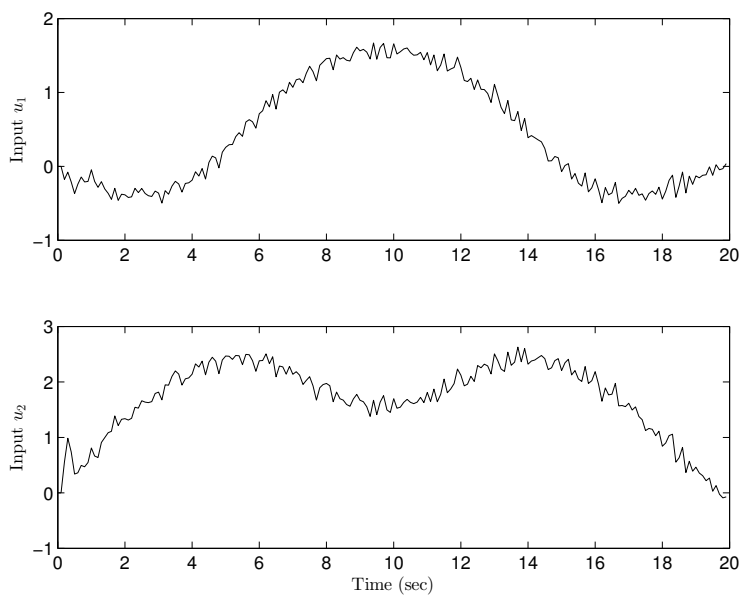

(c) Control signals
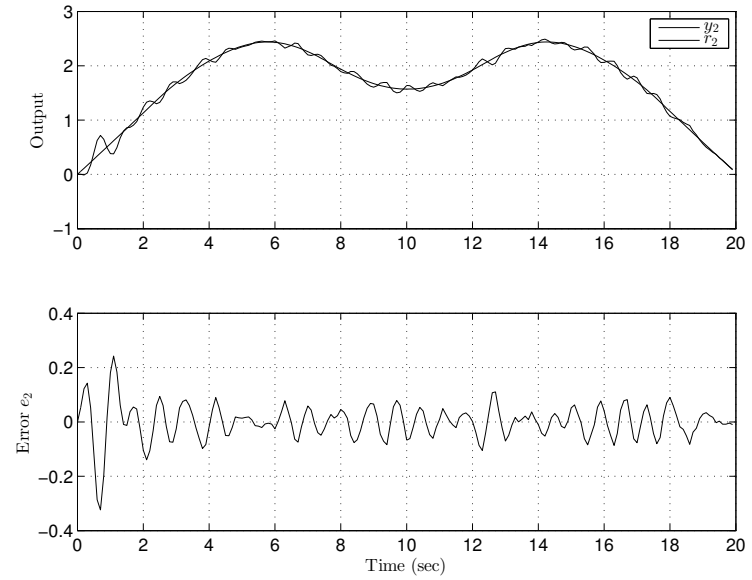

(b) $y_{2}$ and error

Figure 3: Experimental results using the performance parameters in Table 1 except $r_{o b}=100000$. 

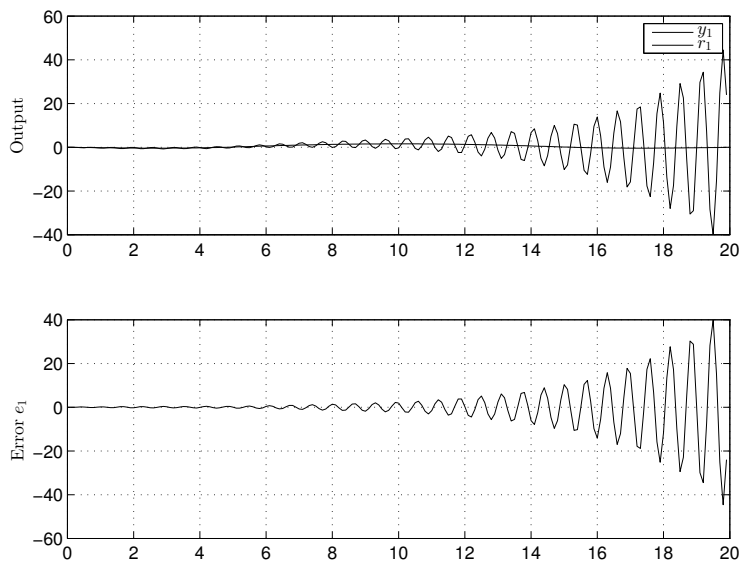

(a) $y_{1}$ and error
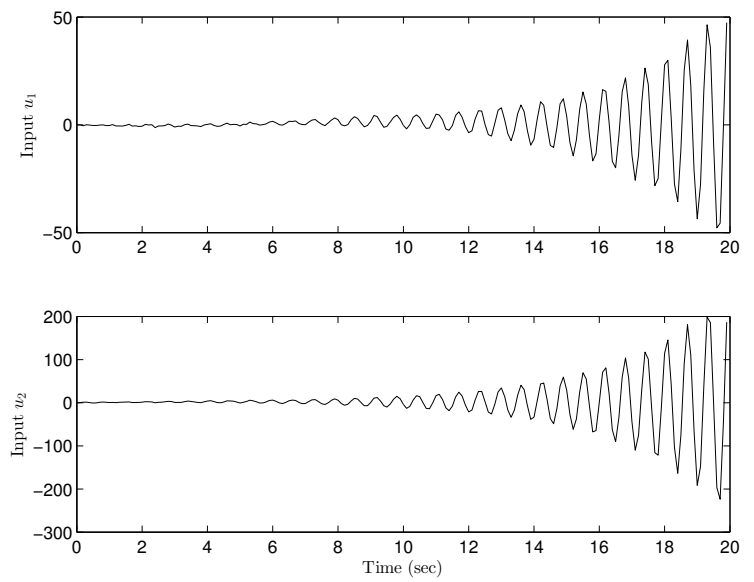

(c) Control signals
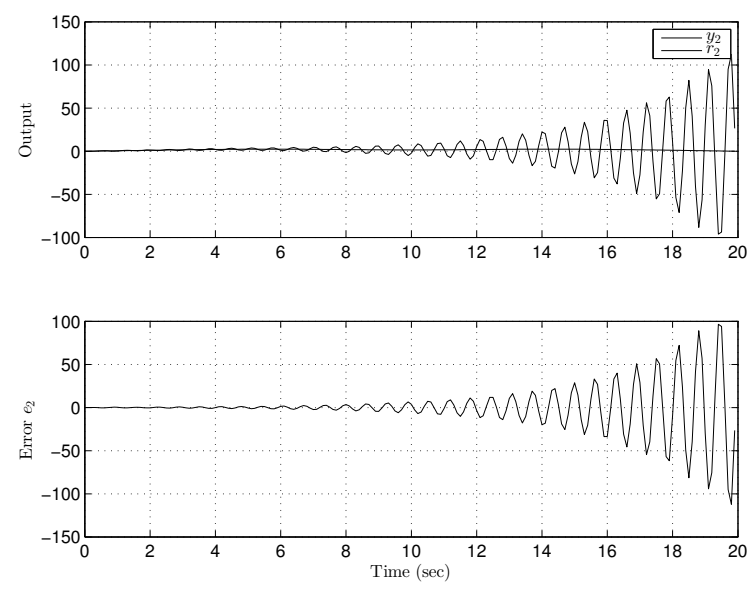

(b) $y_{2}$ and error

Figure 4: Experimental results using the performance parameters in Table 1 except $r_{o b}=1000$. 
Hara, S., Yamamoto, Y., Omata, T., Nakando, M., 1988. Repetitive control system: a new type of servo system for periodic exogenous signals. IEEE Transactions on Automatic Control 33, 659-668.

Bristow, D., Tharayil, M., Alleyne, A., 2006. A survey of iterative learning control. IEEE Control Systems Magazine, $26(3), 96-114$.

Ahn, H., Chen, Y., Moore, K. L. 2007. Iterative Learning Control: Brief Survey and Categorization. IEEE Transactions on Systems, Man and Cybernetics Part C: Applications and Reviews, 37 (6), 1099-1121.

Ahn, H., Chen, Y., Moore, K. L. 2007. Iterative Learning Control: Brief Survey and Categorization. IEEE Transactions on Systems, Man and Cybernetics Part C: Applications and Reviews, 37 (6), 1099-1121.

Lim, I., Hoelzle, D. L., Barton, K. L. 2017. A multi-objective iterative learning control approach for additive manufacturing applications. Control Engineering Practice, 64(11), 7487.

De Roover, D., Bosgra, O. H., Steinbuch, M. 2000. Internal model based design of repetitive and iterative learning controllers for linear multivariable systems. International Journal of Control, 73(10), 914-929.

Freeman, C. T., Ali Alsubaie, M., Cai, Z., Rogers, E., Lewin, P. L. 2013. A common setting for the design of iterative learning and repetitive controllers with experimental verification. Internatinal Journal of Adaptive Control and Signal Processing, 27(3), 230-249.

Bitmead, R. R., Anderson, B. D. O. 1981. Adaptive frequency sampling filters, IEEE Transactions on Circuits and Systems, 28(6) 524-534.

Wang, L., Cluett, W. R. 2000. From Plant Data to Process Control: Ideas for Process Identification and PID Design. Taylor and Francis, London.

Wang, L., Freeman, C. T., Chai, S., Rogers, E. 2012. Experimentally validated repetitive-predictive control of a robot arm with constrainsts. Proceedings of the 2012 American Control Conference, 5495-5500.

Wang, L. 2016. A tutorial on predictive repetitive control. Proceedings of the 2016 Australian Control Conference, $114-119$.

Wang, L., 2009. Model Predictive Control System Design and Implementation using MATLAB. Springer-Verlag.

Moore, K. L., 2012. Iterative Learning Control for Deterministic Systems. Springer-Verlag.

Wang, L., Chai, S., Rogers, E., Freeman, C. T., Nov 2012. Multivariable repetitive-predictive controllers using frequency decomposition. IEEE Transactions on Control Systems Technology 20 (6), $1597-1604$.

Wang, L., Chai, S., Yoo, D., Gan, L., Ng, K., 2015. PID and Predictive Control of Electrical Drives and Power Converters. Wiley-IEEE PRESS.

Wang, L., Freeman, C., Rogers, E., 2013. Repetitive-predictive control system with constraints: design and implementation. Journal of Process Control 23, 956-967.

Wang, L., Freeman, C., Rogers, E., 2016. Predictive iterative learning control with experimental validation. Control Engineering Practice 53, 24-34.

Wang, L., Gawthrop, P., Owens, D. H., Rogers, E., 2010. Switched linear predictive controllers for periodic exogenous signals. International Journal of Control 83 (4), 848-861.

Bohn, C., Cortabarria, A., Härtel, V., Kowalczyk, K., 2004. Active control of engine-induced vibrations in automotive vehicles using disturbance observer gain scheduling. Control Engineering Practice 12 (8), 1029-1039. 
Bodson, M. 2005. Rejection of periodic disturbances of unknown and time-varying frequency. International Journal of Adaptive Control and Signal Processing 19 (2-3), 67-78.

Jia, Q. W. 2009. Disturbance rejection through disturbance observer with adaptive frequency estimation. IEEE Transactions on Magnetics 45 (6), 2675-2678.

White, M. T., Tomizuka, M., Smith, C. 2009. Improved track following in magnetic disk drives using a disturbance observer. IEEE/ASME Transactions on Mechatronics 5 (1), 3-11.

Zhen, Q., and Tomizuka, M. 2008. A disturbance observer approach to detecting and rejecting narrow-band disturbances in hard disk drives. Proceedings of the 10th IEEE International Workshop on Advanced Motion Control (AMC'08). 254-259.

Madoński, R and Herman, P. 2015. Survey on methods of increasing the efficiency of extended state disturbance observers. ISA Transactions 56, 18-27. 